\title{
DETERMINAÇÃO TURBIDIMÉTRICA DO ANTIDEPRESSIVO AMITRIPTILINA EM SISTEMA FIA EXPLORANDO A FORMAÇÃO DO PAR IÔNICO COM LAURIL SULFATO DE SÓDIO
}

\author{
Gustavo Silveira e César Ricardo Teixeira Tarley* \\ Departamento de Ciências Exatas, Universidade Federal de Alfenas, Rua Gabriel Monteiro da Silva, 714, \\ 37130-000 Alfenas - MG, Brasil
}

Recebido em 11/8/07; aceito em 4/4/08; publicado na web em 4/9/08

\begin{abstract}
TURBIDIMETRIC DETERMINATION OF ANTIDEPRESSANT AMITRIPTYLINE IN FIA SYSTEM EXPLOITING THE IONPAIR FORMATION WITH SODIUM LAURYL SULPHATE. The present work purposes the development of an analytical method for amitriptyline determination in pharmaceutical formulations using FIA system. It was based on interaction of amitriplyline with sodium lauryl sulphate in acid medium $(\mathrm{pH} 2.5)$ resulting in the ion-pair formation turbidimetrically detected at $410 \mathrm{~nm}$. The fitting regression equation for range curve from $2.0 \times 10^{-3}$ up to $3.2 \times 10^{-3} \mathrm{~mol} \mathrm{~L}^{-1}$ was found to be analytical signal $=-2.7417+0.1538$ [amitriptyline] $(\mathrm{r}=0.99991)$ with a detection limit of $1.8 \times 10^{-3} \mathrm{~mol} \mathrm{~L}^{-1}$. The precision assessed as relative standard deviation $(\mathrm{n}=$ 10) was found to be 2.40 and $1.94 \%$, for the respective concentration of amitriplyline $2.0 \times 10^{-3}$ and $3.2 \times 10^{-3} \mathrm{~mol} \mathrm{~L}^{-1}$ and the sample throughout was $60 \mathrm{~h}^{-1}$. The accuracy of method was successfully assessed in pharmaceutical formulation after comparison with a reference analytical method.
\end{abstract}

Keywords: flow injection turbidimetry; amitriptyline; sodium lauryl sulphate.

\section{INTRODUÇÃO}

Estudos relacionados ao desenvolvimento de antidepressivos tiveram início na década de 40. A amitriptilina [(dimetilamino-3 propilideno)-5 dibenzo(a, d) ciclo-heptadieno], fármaco sob estudo, é um representante da classe dos antidepressivos tricíclicos (ADTs) resultantes da modificação do núcleo fenotiazínico. A amitriptilina (AMT) é largamente utilizada para o tratamento dos transtornos do humor, especialmente a depressão, e sua estrutura está representada na Figura 1.

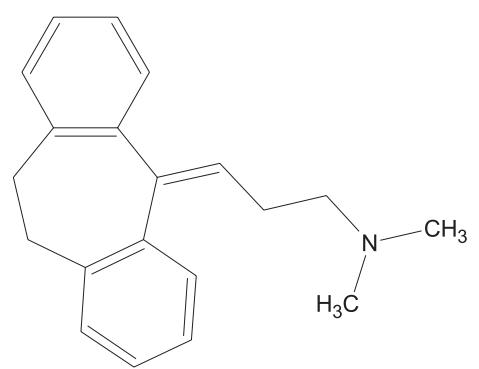

Figura 1. Fórmula estrutural da amitriptilina

Quando usados em doses excessivas, os ADTs podem ser nocivos à saúde, levando até a morte do paciente. Mesmo em doses terapêuticas, há a ocorrência de diversos efeitos colaterais, tais como sonolência e sedação excessiva; confusão mental, secura da boca e visão turva. Sobre o aparelho cardiovascular há o aparecimento de taquicardia e modificações da pressão sanguínea quando administrados em superdosagem. ${ }^{1}$ Por isso, o controle do teor do medicamento administrado é importante para o sucesso do tratamento e minimização dos efeitos colaterais.

A farmacopéia americana recomenda a quantificação por métodos cromatográficos ou titulação com ácido perclórico. ${ }^{2}$ Outros métodos

*e-mail: ctarleyquim@yahoo.com.br cromatográficos também são relatados na literatura para a quantificação de AMT em plasma humano. ${ }^{3}$

Para análise de fármacos, os métodos espectrofotométricos têm sido bastante empregados. Várias estratégias podem ser utilizadas para essa análise. Agentes oxidantes, como Fe (III) e metavanadato de amônio, podem ser utilizados como precursores espectrofotométricos para determinação de diferentes ADTs, embora os métodos propostos requeiram meios ácidos ou longo tempo de reação. ${ }^{4-6}$

A formação de complexos é outra alternativa utilizada para a quantificação de ADTs por espectrofotometria. Podem estar baseados na reação com moléculas orgânicas ou com metais de transição. A quantificação de vários ADTs com cloranil é relatada por Ibrahim et al.. ${ }^{7}$ Abdel-Salam e colaboradores propõem o uso do reagente 2,3 dicloro 5, 6 diciano - p - benzoquinona (DDQ) para a avaliação do teor de ADTs, dentre eles a AMT, em formulações farmacêuticas. ${ }^{8}$ Esses dois agentes espectrofotométricos e ainda iodo foram usados por Bebawy et al. para quantificar fluoxetina e sertralina, dois antidepressivos de nova geração, mostrando a versatilidade desses agentes. ${ }^{9}$ Entretanto, as reações com formação desses complexos ocorrem em meio orgânico, tais como uma mistura de dioxano-etanol, acetonitrila, acetona ou dicloroetano. Além disso, as reações são lentas e requerem aquecimento. A quantificação do complexo de transferência de carga AMT-molibdato de amônio em $\mathrm{H}_{2} \mathrm{SO}_{4}$ concentrado é descrita por Aman et al. ${ }^{10}$

O emprego de pares iônicos, que consistem na interação eletrostática entre o fármaco, protonado, e um contra-íon, carregado negativamente, podendo ser uma molécula orgânica ou um complexo de metal, é a estratégia mais explorada para a análise de antidepressivos. Trata-se de uma possibilidade de análise bastante diversificada, permitindo medidas espectrofotométricas, espectrométricas de absorção atômica ou turbidimétricas. Dentre os metais utilizados, é relatado o uso de complexos de $\mathrm{Ti}(\mathrm{IV}), \mathrm{Nb}(\mathrm{V}), \mathrm{Cr}(\mathrm{III})$ (usado para medidas por absorção atômica) e recentemente, $\mathrm{Mo}(\mathrm{V}) .{ }^{11-15}$ É comum em todos esses métodos a utilização de solventes orgânicos, tais como clorofórmio, álcool butílico, acetona e diclorometano, respectivamente, a fim de solubilizar o par iônico. Além disso, os meios reacionais são 
extremos, com elevada concentração dos reagentes, por exemplo, $\mathrm{HCl}$ e SCN ${ }^{-}$até $10 \mathrm{~mol} \mathrm{~L}^{-1} .{ }^{11}$ Dentre os exemplos de moléculas orgânicas utilizadas na formação de pares iônicos, encontra-se o azul de timol, o cromo azurol S, verde de bromocresol, alaranjado de metila e alizarina S. ${ }^{14,16-20}$ Embora não se utilizem reagentes em elevada concentração, faz-se necessário o uso de solventes orgânicos para extração, condição que implica em maior risco ao operador.

Medidas turbidimétricas têm sido exploradas para a quantificação de diversos tipos de fármacos. Como exemplo, Farhadi et al. tratam de métodos para análise de derivados $\mathrm{N}$-substituídos de fenotiazinas. ${ }^{21}$ Canaes e Fatibello-Filho desenvolveram um método em fluxo para determinação de metilbrometo de homatropina em formulações farmacêuticas, empregando $\mathrm{AgNO}_{3}{ }^{22}$ Calatayud e Pastor também empregaram sistema de análise em fluxo (FIA) com detecção turbidimétrica para quantificação do par iônico AMT-púrpura de bromocresol. ${ }^{23}$

Outros sistemas de análise por injeção em fluxo reportados na literatura requerem extração do composto formado, geralmente feita utilizando dicloroetano ou clorofórmio. ${ }^{17,20}$ Por fim, El-Nashar et al. propõem a quantificação potenciométrica de AMT por injeção em fluxo por meio do emprego de eletrodos íon seletivos construídos pela formação de filmes de fosfotungstato de AMT e fosfomolibdato de AMT com PVC. ${ }^{24}$

Com base nos métodos previamente apresentados, nota-se que a maioria requer aquecimento, longo tempo reacional, necessidade de extração com solventes orgânicos e meios reacionais desfavoráveis. Além disso, constata-se que sistemas FIA apresentam um potencial a ser explorado para determinação de ADTs, principalmente quanto à freqüência analítica e análise sem extração com solventes orgânicos. $\mathrm{O}$ presente método explora medidas turbidimétricas em sistemas em fluxo baseando-se na formação do par iônico AMT-lauril sulfato de sódio, medido em $410 \mathrm{~nm}$. A adoção do surfactante aniônico como substituto das moléculas orgânicas comumente utilizadas e metais responsáveis pela formação de pares iônicos se deve as suas características favoráveis, tais como biodegradabilidade e baixa toxicidade, fatores importantes para o desenvolvimento de métodos analíticos menos agressivos ao meio ambiente que se enquadrem, desta forma, dentro do atual conceito de Química Verde.

\section{PARTE EXPERIMENTAL}

\section{Instrumentação}

As medidas turbidimétricas em sistema FIA foram feitas em um espectrofotômetro de feixe simples Femto, modelo 435 (São PauloBrasil) equipado com uma cela de fluxo de $1,0 \mathrm{~cm}$ de caminho ótico. A aquisição de dados foi realizada por meio de uma interface PCL 711 com programação em macro Visual Basic (Microsoft Excel). Um espectrofotômetro UV-visível Spectrum, modelo SP-2000 UV (Xangai-China) utilizando cela de quartzo de $1 \mathrm{~cm}$ de caminho ótico foi empregado nos estudos de validação do método proposto. Para propulsão dos padrões, amostras e reagentes foi empregada uma bomba peristáltica Ismatec (Zurique-Suíça) de oito canais contendo tubos de Tygon ${ }^{\circledR}$ de diferentes diâmetros e tubos de polietileno 0,8 $\mathrm{mm}$ de diâmetro interno. Um injetor proporcional de três peças feito em Teflon ${ }^{\circledR}$ foi utilizado para introdução dos padrões e amostras no sistema FIA.

\section{Reagentes e soluções}

Cloridrato de amitriptilina ( $\geq 98 \%$, Sigma-Aldrich, Itália) e lauril sulfato de sódio (mínimo de 90\%, Vetec, Brasil) foram utilizados sem prévia purificação. Os demais reagentes empregados foram de grau analítico e todas as soluções foram preparadas em água desionizada em sistema de purificação Milli-Q (Millipore, Brasil). A solução estoque de amitriptilina $1,25 \times 10^{-2} \mathrm{~mol} \mathrm{~L}^{-1}$ foi preparada pela dissolução de 100,0 mg do fármaco em água com posterior transferência para balão volumétrico de $25,0 \mathrm{~mL}$. As demais soluções de amitriptilina foram preparadas por meio de diluições adequadas.

O preparo do lauril sulfato de sódio $\left(2,0 \times 10^{-3} \mathrm{~mol} \mathrm{~L}^{-1}\right)$ tamponado foi realizado pela dissolução de $320,4 \mathrm{mg}$ de lauril sulfato de sódio e de 375,7 mg de ácido tartárico (Isofar, Brasil) em balão volumétrico de 500,0 mL. Antes de aferir o volume do balão volumétrico ajustouse o pH da solução para 2,5 com NaOH 1,0 mol L-1. Para os estudos que envolveram a validação do método foi empregado clorofórmio como solvente extrator (Tedia, Brasil); solução de alaranjado de metila (Isofar, Brasil) 0,02\% (m/v) preparada pela dissolução de 20,0 mg do composto em balão volumétrico de $100,0 \mathrm{~mL}$; tampão tartarato $0,1 \mathrm{~mol} \mathrm{~L}^{-1}, \mathrm{pH} 3,0$, obtido após dissolução de 3,752 g de ácido tartárico em balão de 250,0 mL. Antes da aferição do volume, o pH foi corrigido com $\mathrm{NaOH}$ 1,0 mol L-1. Solução estoque de AMT 1,0 mg $\mathrm{mL}^{-1}$ foi obtida por meio da dissolução de $100,0 \mathrm{mg}$ do fármaco em pequena quantidade de metanol e posterior aferição do volume com balão volumétrico de $100,0 \mathrm{~mL}$.

\section{Sistema de análise por injeção em fluxo}

O sistema de análise por injeção em fluxo por confluência é representado na Figura 2. Alíquotas de $600 \mu \mathrm{L}$ (alça de $120 \mathrm{~cm}$ ) de amitriptilina com concentrações variando de $2,0 \times 10^{-3}$ a $3,2 \times 10^{-3}$ mol L $\mathrm{L}^{-1}$ foram introduzidas no fluxo carregador $\left(\mathrm{H}_{2} \mathrm{O}\right)$ sob uma vazão de $2,75 \mathrm{~mL} \mathrm{~min}^{-1}$. No ponto $\mathrm{X}$, a zona dispersa da amostra recebe por confluência o reagente lauril sulfato de sódio $2,0 \times 10^{-3} \mathrm{~mol} \mathrm{~L}^{-1}$ (vazão de 2,75 $\mathrm{mL} \mathrm{min}^{-1}$ e tamponado em pH 2,5 com tampão tartarato 5,0 x $10^{-3} \mathrm{~mol} \mathrm{~L}^{-1}$ ), promovendo a formação do par iônico insolúvel. A reação se completa através da bobina reacional, $\mathrm{B}$, de $80 \mathrm{~cm}$, com posterior detecção turbidimétrica em $410 \mathrm{~nm}$.

\section{Otimização do sistema de análise por injeção em fluxo}

Para otimizar o sistema de análise por injeção em fluxo foi utilizado um planejamento fatorial fracionário $2^{5-1}$ seguido de um planejamento Doehlert. A adoção de planejamento fracionário em detrimento ao planejamento completo foi efetuada para reduzir o número de experimentos pela metade, sem provocar, no entanto, padrões de confundimento entre os efeitos principais e aqueles relativos aos efeitos de interação. Os fatores investigados foram volume da alça de amostragem, vazão do carregador, concentração do lauril sulfato de sódio e do tampão e o

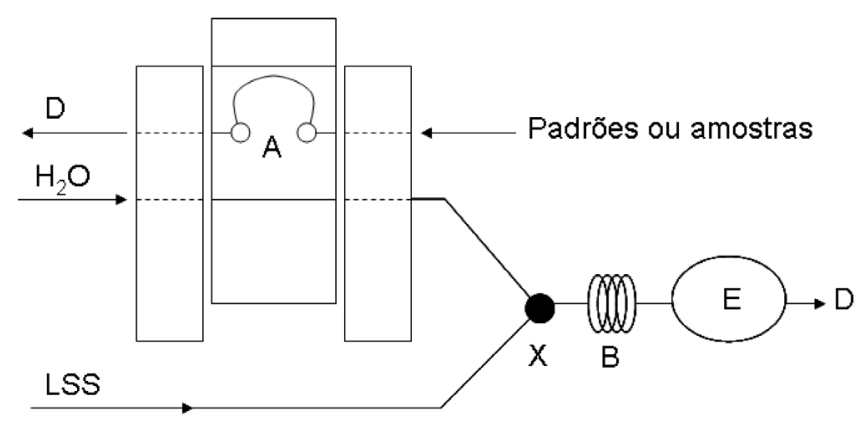

Figura 2. Representação esquemática da configuração por confluência do sistema FIA adotado. Concentração do LSS 2,0 $\times 10^{-3} \mathrm{~mol} \mathrm{~L}^{-1}$, vazão do carregador $\left(\mathrm{H}_{2} \mathrm{O}\right)$ e do LSS de 2,75 $\mathrm{mL} \mathrm{min}^{-1}$. A = alça de amostragem $(600 \mu \mathrm{L})$; $B=$ bobina reacional $(80 \mathrm{~cm}) ; X=$ ponto de confluência $; D=$ descarte 
pH do carregador. Na Tabela 1, estão reunidos os fatores com seus respectivos níveis. Cabe salientar que os níveis escolhidos foram definidos a partir de estudos prévios, justificando, por exemplo, a exclusão da bobina reacional para os níveis dos fatores estudados. Após a etapa de triagem dos fatores e de posse da significância dos fatores no sistema em estudo, empregou-se o planejamento Doehlert a fim de otimizar o método. Todos os experimentos do planejamento fatorial fracionário foram conduzidos com a concentração de AMT fixa em 5,0 x $10^{-3} \mathrm{~mol}$ $\mathrm{L}^{-1}$, ao passo que no planejamento Doehlert a concentração de AMT foi de $2,5 \times 10^{-3} \mathrm{~mol} \mathrm{~L}^{-1}$. Os dados obtidos foram processados por meio do programa STATISTICA (StatSoft, Tulsa, USA).

Tabela 1. Fatores e seus respectivos níveis empregados na construção do planejamento fatorial fracionário $2^{5-1}$

\begin{tabular}{lcc}
\hline Fatores & $\begin{array}{c}\text { Nível } \\
\text { menor }(-)\end{array}$ & $\begin{array}{c}\text { Nível } \\
\text { maior }(+)\end{array}$ \\
\hline Concentração do Tampão $\left(\mathrm{mol} \mathrm{L}^{-1} \times 10^{-3}\right)$ & 5,0 & 10,0 \\
Concentração do LSS $\left(\mathrm{mol} \mathrm{L}^{-1} \times 1^{-3}\right)$ & 5,0 & 10,0 \\
Vazão do Carregador $\left(\mathrm{mL} \mathrm{min}^{-1}\right)$ & 1,70 & 2,40 \\
Volume da Alça $(\mu \mathrm{L})$ & 50 & 150 \\
pH do carregador & 2,5 & 5,0 \\
\hline
\end{tabular}

A vazão do LSS foi igual à vazão empregada para o carregador $\left(\mathrm{H}_{2} \mathrm{O}\right)$

\section{Preparo das formulações farmacêuticas e determinação de amitriptilina}

Foram obtidos comprimidos revestidos de cloridrato de amitriptilina de 4 empresas farmacêuticas, denominadas A, B, C e D. De cada amostra, foram pesados 10 comprimidos e pulverizados em almofariz. Uma quantidade adequada do comprimido foi dissolvida em $10 \mathrm{~mL}$ de ácido sulfúrico $0,5 \mathrm{~mol} \mathrm{~L}^{-1}$, sob agitação ( $\left.10 \mathrm{~min}\right)$, seguida da filtração em papel qualitativo. Posteriormente, corrigiu-se o pH dessa solução com $\mathrm{NaOH}$ 1,0 mol L-1 até o valor de 2,5. As quantidades pesadas de comprimido pulverizado foram feitas de modo a se obter $50,0 \mathrm{~mL}$ de solução de amitriptilina $2,4 \times 10^{-3} \mathrm{~mol} \mathrm{~L}^{-1}$, considerando a dosagem declarada no rótulo do produto.

\section{RESULTADOS E DISCUSSÃO}

Medidas turbidimétricas requerem a manipulação rigorosa das soluções padrão e/ou amostras para obtenção de resultados analíticos precisos, pois qualquer variação no preparo da suspensão pode acarretar na falta de uniformidade do tamanho das partículas formadas e, conseqüentemente, na determinação analítica. Esta dificuldade é pronunciada em sistemas em batelada, porém, bastante minimizada em sistemas de análise por injeção em fluxo..$^{25}$

O diagrama esquemático do sistema em fluxo é mostrado na Figura 2. A influência dos fatores químicos e de fluxo no sistema proposto foi investigada inicialmente a partir de um planejamento fatorial fracionário $2^{5-1}$, cuja combinação dos níveis e os resultados experimentais obtidos encontram-se na Tabela 2. Cabe salientar que a matriz geradora utilizada foi $I=$ abcde, ou seja, os sinais da coluna vertical para o fator $\mathrm{pH}$ foram obtidos multiplicando os sinais dos demais fatores. Para avaliar a significância das estimativas de contraste dos fatores, primeiramente foi calculada a estimativa conjunta da variância $\left(s_{p}^{2}\right)$, com 16 graus de liberdade de acordo com a Equação 1

$s_{p}^{2}=\frac{\left(n_{A}-1\right) s_{A}^{2}+\left(n_{B}-1\right) s_{B}^{2}}{\left(n_{A}-1\right)+\left(n_{B}-1\right)}$

onde, $n$ é o número de repetições em cada ensaio e $\mathrm{s}^{2}$ é a variância obtida em cada ensaio. A partir da Equação 1, obteve-se a estimativa conjunta da variância de 5,82 x 10-4. Fazendo uso da Equação 2 é possível calcular a variância experimental.

$V($ estimativa de contraste $)=V[\bar{R}(+)]+V[\bar{R}(-)]=\frac{s_{p}{ }^{2}}{n}+\frac{s_{p}{ }^{2}}{n}$

onde $n$ (neste caso 16) é o número de respostas com sinais positivos e negativos. Assim, a variância experimental obtida foi de $1,45 \mathrm{x}$ $10^{-4}$. O erro experimental, também conhecido como erro padrão da estimativa, foi calculado extraindo a raiz da variância experimental, sendo, portanto $1,20 \times 10^{-2}$. Empregando a distribuição $t$ de Student, pode-se construir intervalos de confiança para os valores das estimativas e, assim, decidir quais fatores são significativos. Desta

Tabela 2. Combinações dos fatores e resultados do planejamento fatorial fracionário $2^{5-1}$. A matriz geradora empregada foi $I=$ abcde

\begin{tabular}{|c|c|c|c|c|c|c|c|c|}
\hline$N^{o}$ exp. & Conc. Tampão & Conc. LSS & $\mathrm{VC}$ & Volume Alça & $\mathrm{pH}$ & Sinais analíticos & Sinais analíticos médios & Variância \\
\hline 1 & - & - & - & - & + & $0,460 / 0,458$ & 0,459 & $2,00 \times 10^{-06}$ \\
\hline 2 & + & - & - & - & - & $0,366 / 0,375$ & 0,371 & $4,05 \times 10^{-05}$ \\
\hline 3 & - & + & - & - & - & $0,139 / 0,166$ & 0,153 & $3,64 \times 10^{-04}$ \\
\hline 4 & + & + & - & - & + & $0,001 / 0,002$ & 0,002 & $5,00 \times 10^{-07}$ \\
\hline 5 & - & - & + & - & - & $0,376 / 0,341$ & 0,359 & $6,12 \times 10^{-04}$ \\
\hline 6 & + & - & + & - & + & $0,314 / 0,323$ & 0,319 & $4,05 \times 10^{-05}$ \\
\hline 7 & - & + & + & - & + & $0,076 / 0,081$ & 0,079 & $1,25 \times 10^{-05}$ \\
\hline 8 & + & + & + & - & - & $0,187 / 0,186$ & 0,187 & $5,00 \times 10^{-07}$ \\
\hline 9 & - & - & - & + & - & $1,312 / 1,354$ & 1,333 & $8,82 \times 10^{-04}$ \\
\hline 10 & + & - & - & + & + & $0,816 / 0,870$ & 0,843 & $1,46 \times 10^{-03}$ \\
\hline 11 & - & + & - & + & + & $0,205 / 0,211$ & 0,208 & $1,80 \times 10^{-05}$ \\
\hline 12 & + & + & - & + & - & $0,522 / 0,435$ & 0,479 & $3,78 \times 10^{-03}$ \\
\hline 13 & - & - & + & + & + & $0,603 / 0,562$ & 0,583 & $8,41 \times 10^{-04}$ \\
\hline 14 & + & - & + & + & - & $0,870 / 0,876$ & 0,873 & $1,80 \times 10^{-05}$ \\
\hline 15 & - & + & + & + & - & $0,204 / 0,235$ & 0,220 & $4,81 \times 10^{-04}$ \\
\hline 16 & + & + & + & + & + & $0,323 / 0,362$ & 0,343 & $7,60 \times 10^{-04}$ \\
\hline
\end{tabular}

Experimento realizado com concentração de AMT $=5,0 \times 10^{-3} \mathrm{~mol} \mathrm{~L}^{-1}$. 
forma, multiplicando o erro padrão da estimativa pelo valor de $t$ crítico $(2,120)$ com $95 \%$ de confiança e com 16 graus de liberdade, obtém-se o erro padrão de $2,55 \times 10^{-2} .{ }^{26}$ Existe influência significativa do fator quando este apresenta estimativa maior que erro padrão. Portanto, de acordo com a Tabela 3, conclui-se que apenas a concentração do tampão não é significativa no sistema em estudo. Apesar da ausência de influência deste fator, optou-se por fixar a concentração em 5,0 x $10^{-3} \mathrm{~mol} \mathrm{~L}^{-1}$ para os posteriores estudos, uma vez que a adoção do tampão rendeu sinais analíticos com maior repetibilidade.

Tabela 3. Estimativas de contraste para os fatores empregados no planejamento fatorial fracionário $2^{5-1}$

\begin{tabular}{ccc}
\hline $\begin{array}{c}\text { Relações entre as } \\
\text { colunas de sinais }\end{array}$ & Contraste & Estimativas \\
\hline $\mathrm{a}=\mathrm{bcde}$ & $l_{\mathrm{a}} \rightarrow \mathrm{a}+\mathrm{bcde}$ & $l_{a}=0,0028$ \\
$\mathrm{~b}=\mathrm{acde}$ & $l_{b} \rightarrow \mathrm{b}+\mathrm{acde}$ & $l_{b}=-0,4338$ \\
$\mathrm{c}=\mathrm{abde}$ & $l_{\mathrm{c}} \rightarrow \mathrm{c}+\mathrm{abde}$ & $l_{c}=-0,1108$ \\
$\mathrm{~d}=\mathrm{abce}$ & $l_{\mathrm{d}} \rightarrow \mathrm{d}+\mathrm{abce}$ & $l_{d}=0,3693$ \\
$\mathrm{e}=\mathrm{abcd}$ & $l_{\mathrm{e}} \rightarrow \mathrm{e}+\mathrm{abcd}$ & $l_{e}=-0,1423$ \\
$\mathrm{ab}=\mathrm{cde}$ & $l_{\mathrm{ab}} \rightarrow \mathrm{ab}+\mathrm{cde}$ & $l_{a b}=0,0848$ \\
$\mathrm{ac}=\mathrm{bde}$ & $l_{\mathrm{ac}} \rightarrow \mathrm{ac}+\mathrm{bde}$ & $l_{a c}=0,1176$ \\
$\mathrm{ad}=\mathrm{bce}$ & $l_{\mathrm{ad}} \rightarrow \mathrm{ad}+\mathrm{bce}$ & $l_{a d}=0,0457$ \\
$\mathrm{ae}=\mathrm{bcd}$ & $l_{\mathrm{ae}} \rightarrow \mathrm{ae}+\mathrm{bcd}$ & $l_{a e}=0,0416$ \\
$\mathrm{bc}=\mathrm{ade}$ & $l_{\mathrm{bc}} \rightarrow \mathrm{bc}+\mathrm{ade}$ & $l_{b c}=0,1074$ \\
$\mathrm{bd}=\mathrm{ace}$ & $l_{\mathrm{bd}} \rightarrow \mathrm{bd}+\mathrm{ace}$ & $l_{b d}=-0,1619$ \\
$\mathrm{be}=\mathrm{acd}$ & $l_{\mathrm{be}} \rightarrow \mathrm{be}+\mathrm{acd}$ & $l_{b e}=0,0407$ \\
$\mathrm{~cd}=\mathrm{abe}$ & $l_{\mathrm{cd}} \rightarrow \mathrm{cd}+\mathrm{abe}$ & $l_{c d}=-0,1004$ \\
$\mathrm{ce}=\mathrm{abd}$ & $l_{\mathrm{ce}} \rightarrow \mathrm{ce}+\mathrm{abd}$ & $l_{c e}=0,0634$ \\
$\mathrm{de}=\mathrm{abc}$ & $l_{\mathrm{de}} \rightarrow \mathrm{de}+\mathrm{abc}$ & $l_{d e}=-0,0897$ \\
$\mathrm{I}=\mathrm{abcde}$ & $l_{\mathrm{I}} \rightarrow \mathrm{média}+1 / 2(\mathrm{abcde})$ & $l_{I}=0,4253$ \\
\hline
\end{tabular}

a = Concentração do Tampão; $\mathrm{b}=$ Concentração do LSS; c = VC; d $=$ volume da alça e e $=\mathrm{pH}$

A concentração do lauril sulfato de sódio foi o fator mais significativo no sistema estudado, apresentando uma estimativa de contraste de -0,4338. Este valor negativo implica que ao se elevar o nível deste fator $\left(5,0\right.$ para $\left.10,0 \times 10^{-3} \mathrm{~mol} \mathrm{~L}^{-1}\right)$ há um decréscimo na resposta analítica. Este comportamento sugere que, devido à elevada concentração do surfactante, possivelmente há a formação de micelas capazes de solubilizar o par iônico amitriptilina-lauril sulfato de sódio, com a conseqüente redução no sinal analítico. ${ }^{21} \mathrm{O}$ segundo fator mais importante foi o volume da alça de amostragem. Neste caso, a estimativa de contraste foi positiva $(0,3693)$, revelando que incrementos no volume de amostragem promovem aumento no sinal analítico. A estimativa de contraste obtida para o fator $\mathrm{pH}(-0,1423)$ coaduna-se com a reação química para a formação do par iônico, pois em valores baixos de $\mathrm{pH}$ a molécula de amitriptilina está protonada $(\mathrm{pKa}=9,4)$, podendo estabelecer uma atração eletrostática com o surfactante.

Por fim, o fator vazão do carregador apresentou estimativa de contraste negativa $(-0,1108)$ dentro do domínio experimental $(1,70$ a 2,40 $\mathrm{mL} \mathrm{min}^{-1}$ ), sugerindo o emprego de vazões menores que $2,40 \mathrm{~mL}$ $\min ^{-1}$ na otimização. O resultado observado deve-se ao efeito do tempo de residência sobre a formação da suspensão. A menor vazão do carregador resulta num maior tempo para a nucleação, levando ao aumento do sinal analítico.

Após a etapa de triagem dos fatores empregou-se a matriz de Doehlert a fim de otimizar o método. A matriz de Doehlert compreende um planejamento com número de experimentos igual a $k^{2}+k+p c$, em que $k$ é o número de fatores e $p c$ é o número de experimentos no ponto central. Uma característica importante do planejamento Doehlert refere-se ao número de níveis empregados para cada fator. Empregando dois fatores, por exemplo, o número de níveis é 3 e 5, o que permite avaliar com maior número de níveis para aqueles fatores considerados mais importantes. ${ }^{27}$ Além disso, este tipo de planejamento necessita de um menor número de experimentos em relação a outros planejamentos, como o planejamento composto central sendo, assim, mais eficiente.

Foram construídas duas matrizes de planejamento Doehlert. A primeira contém os fatores concentração do lauril sulfato de sódio e volume de amostragem (Tabela 4), uma vez que a interação destes fatores é bastante significativa. Na construção da segunda matriz foram empregados os fatores pH e vazão do carregador (Tabela 5). De acordo com a Figura 3A, obtida a partir da matriz de Doehlert (Tabela 4) e mantendo fixa a vazão do carregador em 2,00 mL $\min ^{-1}$ e pH 3,0, observa-se um ponto de cela. ${ }^{28}$ Como constatado, nota-se a presença de um valor ótimo para a concentração do lauril sulfato de sódio $\left(2,0 \times 10^{-3} \mathrm{~mol} \mathrm{~L}^{-1}\right)$, ao passo que elevados sinais analíticos podem ser obtidos com volumes de amostragem de 1000 e $600 \mu \mathrm{L}$. Assim, empregou-se a concentração de lauril sulfato de sódio de $2,0 \times 10^{-3} \mathrm{~mol} \mathrm{~L}^{-1}$ e o volume de amostragem de $600 \mu \mathrm{L}$. Em seguida, com os dados da Tabela 5, obteve-se a superfície de resposta mostrada na Figura 3B, a qual foi construída sob as condições previamente otimizadas de lauril sulfato de sódio e volume de amostragem. Verificou-se, como esperado, que o sinal analítico aumenta linearmente com a diminuição do $\mathrm{pH}$ do carregador. Experimentos com valores de $\mathrm{pH}$ abaixo de 2,5 não foram conduzidos para evitar a hidrólise do lauril sulfato de sódio e, portanto, fixou-se o pH em 2,5 para os posteriores estudos. ${ }^{29} \mathrm{Com}$ relação à vazão do carregador, nota-se, de acordo com a Figura 3B, que os valores inferior $\left(1,90 \mathrm{~mL} \mathrm{~min}{ }^{-1}\right)$ e superior $\left(2,75 \mathrm{~mL} \mathrm{~min}^{-1}\right)$ podem ser empregados, resultando em elevados sinais analíticos, desde que o meio seja ácido ( $\mathrm{pH} 2,5)$. Tal como observado na triagem dos

Tabela 4. Matriz de Doehlert para as variáveis [LSS] e comprimento da alça de amostragem

\begin{tabular}{|c|c|c|c|}
\hline \multicolumn{4}{|c|}{$\mathrm{N}^{o} \exp [\mathrm{LSS}]\left(\mathrm{mol} \mathrm{L}^{-1} \times 10^{-3}\right)$ Volume de alça $(\mu \mathrm{L})$ Sinais analíticos } \\
\hline 1 & 2,5 & 800 & $0,461 / 0,486$ \\
\hline 2 & 5,0 & 800 & $0,141 / 0,128$ \\
\hline 3 & 3,7 & 1000 & $0,221 / 0,246$ \\
\hline 4 & 0,01 & 800 & $0,001 / 0,001$ \\
\hline 5 & 1,2 & 600 & $0,903 / 0,926$ \\
\hline 6 & 3,7 & 600 & $0,224 / 0,221$ \\
\hline 7 & 1,0 & 1000 & $0,945 / 0,934$ \\
\hline
\end{tabular}

Experimentos realizados com concentração de AMT $2,5 \times 10^{-3} \mathrm{~mol}$ $\mathrm{L}^{-1}$, em pH 3,0 e vazão do carregador e $\mathrm{LSS}=2,00 \mathrm{~mL} \mathrm{~min}{ }^{-1}$.

Tabela 5. Matriz de Doehlert para as variáveis pH e vazão do carregador

\begin{tabular}{lccc}
\hline $\mathrm{N}^{\mathrm{o}} \exp$ & $\mathrm{pH}$ & $\mathrm{VC}\left(\mathrm{mL} \mathrm{min}^{-1}\right)$ & Sinais analíticos \\
\hline 1 & 3,7 & 2,32 & $0,867 / 0,844$ \\
2 & 5,0 & 2,32 & $0,770 / 0,761$ \\
3 & 4,4 & 2,75 & $0,952 / 0,972$ \\
4 & 2,5 & 2,32 & $1,027 / 1,023$ \\
5 & 3,1 & 1,90 & $0,949 / 0,948$ \\
6 & 4,4 & 1,90 & $0,849 / 0,875$ \\
7 & 3,1 & 2,75 & $0,939 / 0,973$ \\
\hline
\end{tabular}

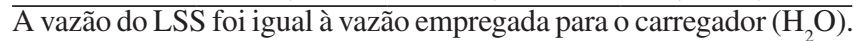
Concentração de AMT 2,5 x $10^{-3} \mathrm{~mol} \mathrm{~L}^{-1}$. 


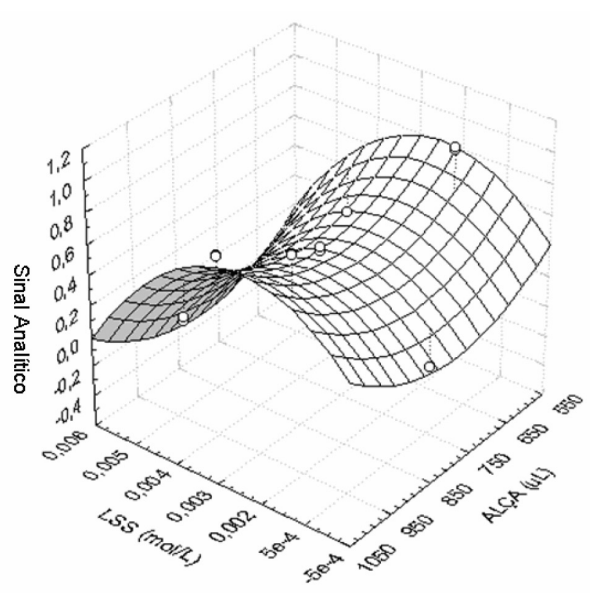

(A)

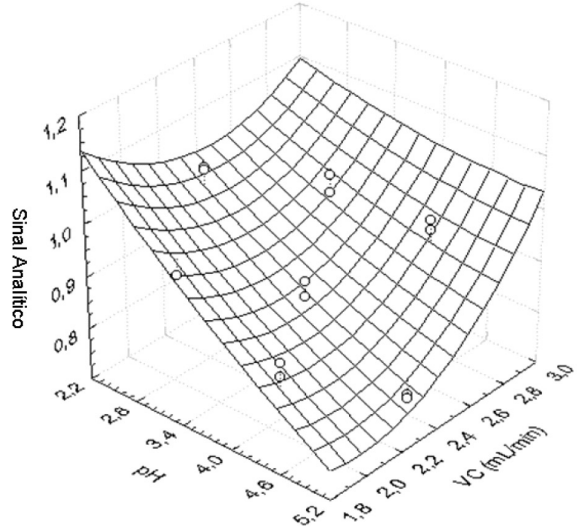

(B)

Figura 3. Superficie de resposta obtida a partir da matriz de Doehlert. (A) superfície construída com dados da Tabela 4 fixando o pH em 3,0 e a vazão do carregador em 2,00 $\mathrm{mL} \mathrm{min}^{-1}$; (B) superfície construída com dados da Tabela 5 fixando a alça em $600 \mu \mathrm{L}$ e a concentração de LSS em 2,0 x 10-3 mol L-2

fatores, os experimentos realizados em vazões menores favorecem a formação da suspensão (maior tempo para nucleação). Entretanto, a explicação para os elevados sinais analíticos em valores maiores de vazão possivelmente esteja associada com o elevado volume de amostragem $(600 \mu \mathrm{L})$, o qual é consideravelmente maior que aqueles empregados na triagem $(50$ a $150 \mu \mathrm{L})$. Com o uso de volume de amostragem de $600 \mu \mathrm{L}$ foi atingido um sinal analítico constante (steady state). Assim, o emprego de vazões baixas e elevadas, provoca, respectivamente, apenas alargamento e estreitamento do sinal analítico, ao passo que a altura de sinal praticamente é inalterada devido à pequena diferença entre as vazões $\left(1,90\right.$ até $\left.2,75 \mathrm{~mL} \mathrm{~min} \mathrm{~m}^{-1}\right)$. Desta forma, optou-se por empregar a vazão de $2,75 \mathrm{~mL} \mathrm{~min}^{-1}$ a fim de obter maior freqüência analítica.

Para finalizar a otimização do método, avaliou-se o efeito do comprimento da bobina reacional no sistema (50 a $90 \mathrm{~cm}$ ) (Figura 4). É interessante notar que a inserção da bobina reacional se tornou fundamental no intuito de melhorar o desempenho do sistema. $\mathrm{O}$ sinal analítico aumenta gradualmente até o valor de $80 \mathrm{~cm}$, tornando evidente o efeito da nucleação do precipitado sobre o sinal analítico. O efeito da dispersão no sistema só se torna importante após esse comprimento da bobina reacional. Assim sendo, a melhor relação entre o tempo de residência na nucleação do precipitado e a dispersão é obtida com uma bobina reacional de $80 \mathrm{~cm}$, sendo este o comprimento da bobina empregado neste trabalho.

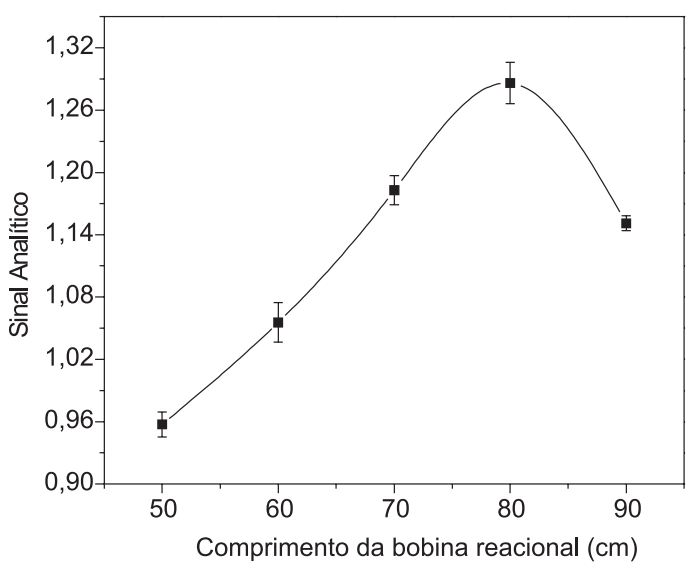

Figura 4. Efeito do comprimento da bobina sobre o sinal analítico

\section{Estudo de interferentes no método proposto}

A influência de concomitantes no sistema de injeção em fluxo foi avaliada por meio da comparação do sinal analítico obtido para uma solução padrão de amitriptilina $\left(785 \mathrm{mg} \mathrm{L}^{-1}\right)$ com aquela contendo concomitantes em potencial em diferentes concentrações. Percentuais de recuperação do sinal analítico variando de 95 a $105 \%$ para a concentração do padrão de amitriptilina demonstram a não influência dos concomitantes, uma vez que o erro tolerado em análises farmacêuticas é de $\pm 5 \% .{ }^{25}$ Foram empregados como concomitantes excipientes comumente usados em formulações farmacêuticas: lactose, dextrose, glicose, amido e estearato de magnésio. Para os excipientes lactose, dextrose e glicose o teste de interferência foi conduzido pela solubilização de amitriptilina com o concomitante em água. Já para amido e estearato de magnésio, o procedimento adotado consistiu da homogeneização de 20,0 mg de amitriptilina e 100,0 mg do excipiente, seguida pela filtração a vácuo com $25 \mathrm{~mL}$ de água em funil de vidro sinterizado. A solução filtrada foi posteriormente introduzida no sistema de análise em fluxo. De acordo com a Tabela 6, verifica-se que os sacarídeos não interferiram na quantificação do fármaco, mesmo em concentrações 60 vezes maiores que do analito. É importante ressaltar que os teores destes excipientes nos produtos farmacêuticos são inferiores aos testados neste estudo. Com relação ao concomitante amido, constatou-se também não haver interferência no sinal analítico, já que a recuperação foi de $104,7 \%$. Por outro lado, o estearato de magnésio provocou interferência, rendendo recuperação de $73,8 \%$. A redução significativa do sinal analítico pode ser devida à solubilização parcial do concomitante durante o procedimento de filtração permitindo sua reação com a amitriptilina e, conseqüentemente, reduzindo a quantidade do analito para reagir com o lauril sulfato de sódio. Apesar desta interferência, a relação testada analito/ concomitante $(1: 4 \mathrm{~m} / \mathrm{m})$ é inferior àquelas utilizadas em formulações

Tabela 6. Estudo da relação analito/concomitante no percentual de recuperação do sinal analítico para amitriptilina*

\begin{tabular}{lcc}
\hline Concomitantes & $\begin{array}{c}\text { Relação analito/ } \\
\text { concomitante }\end{array}$ & $\begin{array}{c}\text { Recuperação }(\%) \text { do } \\
\text { sinal analítico para } \\
\text { amitriptilina }\end{array}$ \\
\hline Dextrose & $1 / 1,1 / 10,1 / 60$ & 99,$3 ; 99,3 ; 95,9$ \\
Lactose & $1 / 1,1 / 10,1 / 60$ & 99,$2 ; 99,3 ; 94,7$ \\
Glicose & $1 / 1,1 / 10,1 / 60$ & 100,$2 ; 99,5 ; 97,4$ \\
\hline
\end{tabular}

*Concentração de amitriptilina $785 \mathrm{mg} \mathrm{L}^{-1}$ 
farmacêuticas, o que permitirá, conforme será demonstrado, aplicar o método para quantificação de amitriptilina nas referidas amostras.

\section{Figuras de mérito e aplicação do método}

O sistema de análise por injeção em fluxo com detecção turbidimétrica desenvolvido neste trabalho é totalmente dependente da concentração do LSS pois, como mencionado, o excesso de LSS em relação à amitriptilina resulta na formação de micelas, responsáveis pela solubilização do par iônico e conseqüente redução do sinal analítico. Como observado na Figura 5, há duas regiões lineares da curva analítica, a primeira onde há excesso de LSS e a segunda onde há excesso de amitriptilina. Adotou-se a segunda região da curva $\left(2,0 \times 10^{-3}\right.$ a $\left.3,2 \times 10^{-3} \mathrm{~mol} \mathrm{~L}^{-1}\right)$ devido à maior sensibilidade e linearidade [sinal analítico $=-2,7417+0,1538$ [amitriptilina $\mathrm{x}$ $\left.\left.10^{-4} \mathrm{~mol} \mathrm{~L}^{-1}\right](\mathrm{r}=0,99991)\right]$. O limite de detecção do método $(1,8 \mathrm{x}$ $10^{-3} \mathrm{~mol} \mathrm{~L}^{-1}$ ) foi calculado a partir da intersecção das duas equações lineares, similarmente ao procedimento empregado em medidas potenciométricas. ${ }^{30} \mathrm{~A}$ precisão do método foi avaliada em termos de repetibilidade $(n=10)$ para concentrações de $2,0 \times 10^{-3}$ e $3,2 \times 10^{-3}$ mol L ${ }^{-1}$, rendendo os respectivos desvios padrão relativos, $2,40 \mathrm{e}$

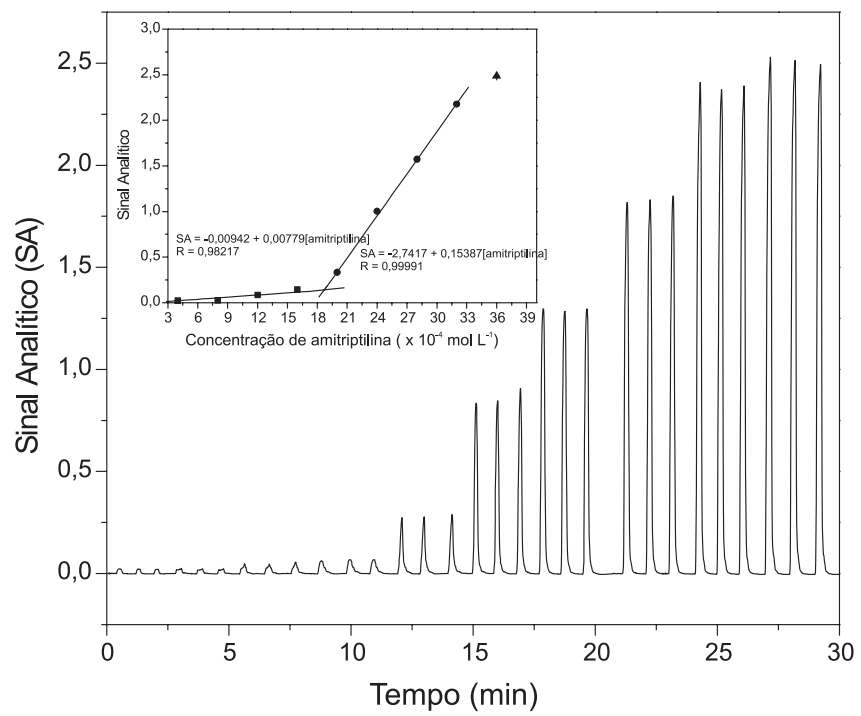

Figura 5. Fiagrama e curva de calibração para determinação de amitriptilina. Sinais obtidos em triplicata mediante injeção de padrões de amitriptilina $\left(2,0 \times 10^{-3} ; 2,4 \times 10^{-3} ; 2,8 \times 10^{-3} ; 3,2 \times 10^{-3}\right.$ e $\left.3,6 \times 10^{-3} \mathrm{~mol} \mathrm{~L}^{-1}\right)$ para segunda região da curva. Condições experimentais conforme descrito na parte experimental

Tabela 7. Determinação de amitriptilina em amostras de formulações farmacêuticas empregando o método proposto e o método de referência

Cloridrato de Amitriptlina (mg/comprimido)

Valor encontrado Valor encontrado

\begin{tabular}{lccc} 
Amostras & Valor rotulado & $\begin{array}{c}\text { Valor encontrado } \\
\text { pelo método } \\
\text { turbidimétrico FIA }\end{array}$ & $\begin{array}{c}\text { Valor encontrado } \\
\text { pelo método de } \\
\text { referência }\end{array}$ \\
\hline (A) & 75,0 & $73,4 \pm 4,3$ & $75,9 \pm 2,8$ \\
(B) & 75,0 & $74,6 \pm 2,9$ & $78,9 \pm 0,9$ \\
(C) & 75,0 & $71,6 \pm 1,2$ & $72,6 \pm 2,1$ \\
(D) & 25,0 & $23,2 \pm 0,9$ & $24,7 \pm 0,4$ \\
\hline
\end{tabular}

$\mathrm{n}=3$; valor de $t$ calculado $=3,147$; valor de $t$ tabelado $=3,182$, com $95 \%$ de confiança.
1,94\%. O método proposto apresentou frequiência analítica de cerca de 60 determinações por hora.

Para avaliar a aplicabilidade do método foram analisadas as quatro amostras A, B, C e D, tratadas conforme descrito na parte experimental e os resultados foram comparados com um método de referência publicado na literatura (Tabela 7). ${ }^{19} \mathrm{O}$ método de referência é baseado na formação do par iônico entre a amitriptilina e alaranjado de metila com posterior extração e solubilização do par iônico em clorofórmio e determinação no comprimento de onda de $420 \mathrm{~nm}$. De acordo com os resultados e, aplicando o teste $t$ pareado, com um nível de confiança de $95 \%$, constata-se que o método proposto não apresenta diferença estatística significativa em relação ao método de referência, confirmando assim a exatidão para as amostras analisadas. ${ }^{31}$

\section{CONCLUSÃO}

Os métodos analíticos comumente empregados para a determinação de amitriptilina são caracterizados pelo uso de solventes orgânicos, ácidos concentrados e, principalmente, pelo longo tempo de análise. Como pôde ser visto, o método proposto reúne várias vantagens, incluindo simplicidade, reduzido consumo de amostras/reagentes devido à mecanização da análise, baixo custo e elevada frequiência analítica, podendo ser aplicado em laboratórios de controle de qualidade. Também, deve-se destacar que o método se enquadra no conceito de Química Verde, pois é empregado o surfactante lauril sulfato de sódio como reagente, ao contrário dos métodos usuais que fazem uso de reagentes muitas vezes tóxicos, seguidos por extração com solventes orgânicos.

De acordo com a revisão da literatura, nota-se que os métodos já existentes de quantificação de amitriptilina possuem limites de detecção e quantificação bastante baixos, ${ }^{5,11-13,19,23}$ bem como ampla faixa linear. Embora essas características analíticas não tenham sido obtidas no método proposto, elas não constituem uma limitação, pois os teores do princípio ativo nas formulações farmacêuticas são suficientes para o preparo de amostras com concentrações adequadas à leitura e à faixa linear, conforme demonstrado pela aplicação satisfatória do método. Finalmente, o método apresentou precisão e exatidão satisfatórias permitindo sua aplicação em diferentes formulações farmacêuticas para quantificação de amitriptilina.

\section{AGRADECIMENTOS}

À FAPEMIG, CNPq, CAPES e ao programa PROBIC/CNPq Unifal-MG pela bolsa de iniciação científica concedida ao acadêmico G. Silveira. Também, os autores agradecem à empresa farmacêutica Medley pelo fornecimento de amostras e aos assessores pelas valiosas sugestões.

\section{REFERÊNCIAS}

1. Silva, P.; Farmacologia, $5^{\text {a }}$ ed., Guanabara Koogan S.A.: Rio de Janeiro, 1998, cap. 32.

2. The United States Pharmacopeia - The National Formulary - USP 24, NF - 19, 2000, p. 122-123.

3. Kollroser, M.; Schober, C.; Therapeutic Drug Monitoring 2002, 24, 537.

4. Nagaraja, P.; Silwadi, M. F.; Syed, A. A.; Mikrochim. Acta 2000, 135, 185.

5. Revanasiddappa, H. D.; Manju, B.; Eur. J. Pharm. Sci. 1999, 9, 221.

6. Misiuk, W.; J. Pharm. Biomed. Anal. 2000, 22, 189.

7. Ibrahim, S. A.; Issa, A. S.; Salam, M. A. A.; Mahrous, M. S.; Talanta 1983, 30, 531 .

8. Abdel-Salam, M.; Issa, A. S.; Mahrous, M.; Abdel-Hami, M. E.; Anal. Lett. 1985, 18, 1391. 
9. Bebawy, L. I.; El-Kousy, N.; Suddik, J. K.; Shokry, M.; J. Pharm. Biomed. Anal. 1999, 21, 133.

10. Aman, T.; Kazi, A. A.; Hussain, M. I.; Firdous, S.; Khan, I. U.; Anal. Lett. 2000, 33, 2477.

11. Misiuk, W.; Tarasiewicz, M.; Anal. Lett. 1998, 31, 1197.

12. Misiuk, W.; Puzanowska-Tarasiewicz, H.; Anal. Lett. 2002, 35, 1163.

13. Elnemma, E. M.; El Zawawy, F. M.; Hassan, S. S. M.; Mikrochim. Acta 1993, $110,79$.

14. Puzanowska-Tarasiewicz, H.; Kuzmicka, L.; Kleszczewska, E.; $J$. Pharm. Biomed. Anal. 2001, 26, 1.

15. Mohamed, G. G.; El-Dien, F. A. N.; Khalil, S. M.; Mohamed, N. A.; Spectrochim. Acta, Part A 2006, 65, 1221.

16. Nagaraja, P.; Silwadi, M. F.; Syed, A. A.; Acta Pharm. 2002, 52, 289.

17. Starczewska, B.; Halaburda, P.; Kojlo, A.; J. Pharm. Biomed. Anal. 2002, 30, 553 .

18. Starczewska, B.; Mielech, K.; J. Pharm. Biomed. Anal. 2000, 23, 243.

19. El-Dien, F. A. F. N.; Mohamed, G. G.; Mohamed, N. A.; Spectrochim. Acta, Part A 2006, 65, 20.

20. Pérez-Ruiz, T.; Martinez-Lozano, C.; Sanz, A.; Alonso, C.; Talanta 1994, 41, 1523.
21. Farhadi, K.; Savojbolaghi, A. K.; Farajzadeh, M.; Maleki, R.; Anal. Lett. 2003, 36, 2183

22. Canaes, L. S.; Fatibello-Filho, O.; Quim. Nova 2006, 29, 1237.

23. Calatayud, M.; Pastor, M.; Anal. Lett. 1990, 23, 1371.

24. El-Nashar, R. M.; Ghani, N. T. A.; Bioumy, A. A.; Microchem. J. 2004, $78,107$.

25. Canaes, L. S.; Dissertação de Mestrado, Universidade Federal de São Carlos, Brasil, 2004.

26. Barros Neto, B.; Scarminio, I. S.; Bruns, R. E.; Como Fazer Experimentos: Pesquisa e Desenvolvimento na Ciência e na Indústria, Ed. da Unicamp: Campinas, 2001, cap. 3.

27. Teófilo, R. F.; Ferreira, M. M. C.; Quim. Nova 2006, 29, 338.

28. Tarley, C. R. T.; Segatelli, M. G.; Kubota, L. T.; Talanta 2006, 69, 259.

29. Handbook of Pharmaceutical Excipients, $2^{\text {nd }}$ ed., American Pharmaceutical Association, Washington e The Pharmaceutical Press, Royal Pharmaceutical Society of Great Britain: London, 1994.

30. Buck, R. P.; Lindner, E.; Pure Appl. Chem. 1994, 66, 2527.

31. Harris, D. C.: Análise Química Quantitativa, 6 ${ }^{\mathrm{a}}$ ed, LTC - Livros Técnicos e Científicos Ed. S. A.: Rio de Janeiro, 2005, cap. 4. 